\title{
Long-Term Outcome of Patients Undergoing Microscopic Lumbar Spinous Process-Splitting Laminectomy
}

Hiroshi Nomura*, Yoshikazu Yanagisawa and Junichi Arima

Department of Orthopedic Surgery, Hiroshima Red Cross Hospital \& Atomic-bomb Survivors Hospital, Hiroshima, Japan

\begin{abstract}
Microscopic lumbar spinous process-splitting laminectomy (LSPSL) has been previously reported as the least invasive surgery for patients with lumbar spinal canal stenosis (LSS). An 18-mm diameter tubular retractor was inserted between the split spinous processes and complete microscopic laminectomy performed in single-level decompressions. A cervical retractor was used to open the caudal parts of the individual halves of the spinous process through a single incision in multi-level decompressions. In this study, we examined long-term outcomes of patients undergoing microscopic LSPSL.
\end{abstract}

Overall, 119 patients with a follow-up longer than 24 months were included in the study. All patients were divided into two groups: 1) the slip group, which included patients with spondylolisthesis-type LSS, and 2) the nonslip group, which included patients with spondylosis-type LSS. The clinical outcomes were evaluated using the Japanese Orthopedic Association score and improvement rate. The slip and instability rates were measured using radiographic imaging findings of the sagittal plane in patients in the slip group. Computed tomography was used to assess bony union of the split spinous process. Magnetic resonance imaging was used to evaluate signal changes of the multifidus muscle at different levels of LSPSL decompression.

After LSPSL, pre-operative symptoms were improved with a significant difference in the Japanese Orthopedic Association score. The overall improvement rate was $62.2 \%$; however, it was $48.6 \%$ in patients older than 79 years. No significant difference was seen in the slip or instability rate before and after LSPSL. The bony union rate of the split spinous process at the site between the process and the vertebral arch was $79.5 \%$. Post-operative magnetic resonance imaging findings demonstrated a slight amount of fat infiltration in the multifidus muscles after LSPSL. In conclusion, the long-term outcome of patients undergoing LSPSL is satisfactory for a minimally invasive decompression surgery.

Keywords: Lumbar spinal stenosis; Lumbar spinous processsplitting laminectomy; Tubular retractor; METRx Microdiscectomy system; Minimally invasive surgery

\section{Introduction}

Lumbar spinal stenosis (LSS) is caused by a gradual narrowing of the spinal canal. It is associated with compression of the dural tube and spinal nerve root, and degenerative alteration in the lumbar spine. Various minimally invasive laminectomies have recently been introduced in patients with LSS, including bilateral decompression via the unilateral approach using a microendoscope and tubular retractor [1-3], lumbar muscle-preserving interlaminar decompression [4], and lumbar spinous process-splitting laminectomy (LSPSL) [5-7].

Of these procedures, we previously reported the clinical outcomes of patients undergoing microscopic LSPSL and found that it was the most minimally invasive surgery for patients with LSS [8]. In particular, it allowed the preservation of paravertebral muscles while minimizing their detachment from the spinous process and posterior supporting structures [8]. For single-level decompressions, we made a skin incision of approximately $20 \mathrm{~mm}$, inserted a tubular retractor from the METRx ${ }^{\mathrm{TM}}$ microdiscectomy system (Medtronic Sofamor Danek Co., Ltd.) between the split spinous processes, and completed the microscopic laminectomy under a microscope. Because the split process was reconstructed after decompression using a simple method, the spinous process and interspinous ligament were preserved. For multi-level decompressions, a cervical retractor (Trimline ${ }^{\circledR}$, Medtronic Sofamor Danek Co., Ltd.) was used to open the caudal parts of the individual halves of the spinous process through a single incision to avoid skin trouble without several small incisions for insertion the tubular retractor. In our previous study, we found that LSPSL results in excellent clinical improvement in patients with LSS. Furthermore, we compared two groups of patients with excellent or fair clinical outcomes, focusing on factors that may influence clinical improvement, including age at the time of surgery, number of decompression levels, operative time, and existence of slipped vertebra; however, no apparent factors was found.

To further distinguish the prognosis of patients with LSPSL, we examined long-term outcomes in patients undergoing LSPSL. In our previous study, we selected patients with follow-up periods greater than 12 months and the mean overall follow-up period was $31 \pm 15$ months. In the current study, we evaluated clinical outcomes in patients who underwent LSPSL with a follow-up period greater than 24 months to identify more stable outcomes. In particular, we focused on the impact of patients' age at the time of surgery on clinical improvement.

\section{Materials and Methods}

This study was approved by the Ethics Committee of Hiroshima Red Cross Hospital and Atomic-bomb Survivors Hospital. Between July 2005 and September 2012, 196 patients with LSS underwent LSPSL at the Hiroshima Red Cross Hospital \& Atomic-bomb Survivors Hospital. Of these patients, 119 (72 men, 47 women) with a followup greater than 24 months were included in this study (follow-up rate was $61 \%)$. The mean age of patients at the time of surgery was $72 \pm$

*Corresponding author: Hiroshi Nomura, MD, PhD, Department of Orthopedic Surgery, Hiroshima Red Cross Hospital \& Atomic-bomb Survivors Hospital, 1-9-6 Senda-Machi, Naka-Ku, Hiroshima 730-8619, Japan,Tel: +81-82-241-3111; Fax: +81-82-246-0676; E-mail: hiroshi20052002@yahoo.co.jp

Received October 22, 2016; Accepted November 22, 2016; Published November 24, 2016

Citation: Nomura H, Yanagisawa Y, Arima J (2016) Long-Term Outcome of Patients Undergoing Microscopic Lumbar Spinous Process-Splitting Laminectomy. J Spine 5: 343. doi: 10.4172/2165-7939.1000343

Copyright: ( $) 2016$ Nomura H, et al. This is an open-access article distributed under the terms of the Creative Commons Attribution License, which permits unrestricted use, distribution, and reproduction in any medium, provided the original author and source are credited. 
Citation: Nomura H, Yanagisawa Y, Arima J (2016) Long-Term Outcome of Patients Undergoing Microscopic Lumbar Spinous Process-Splitting Laminectomy. J Spine 5: 343. doi: 10.4172/2165-7939.1000343

Page 2 of 5

7.4 years (range, $44-93$ years), mean overall follow-up period was 45 \pm 17.5 months (range, 24-98 months), and average decompression level was $1.6 \pm 0.6(1$ level, $n=55 ; 2$ levels, $n=58 ; 3$ levels, $n=6)$. The 119 patients were divided into 2 groups based on clinical symptoms: 1) patients with intermittent claudication $(n=91)$, and 2) patients with radiculopathy $(\mathrm{n}=28)$. The patients were also divided into two groups based on radiographic imaging classification: 1) the slip group $(n=58)$, comprising patients with spondylolisthesis-type LSS with vertebral body slippage; and 2) the nonslip group $(\mathrm{n}=61)$, comprising patients with spondylosis-type LSS without vertebral body slippage or LSS from central protrusion of lumbar disc herniation (Table 1).

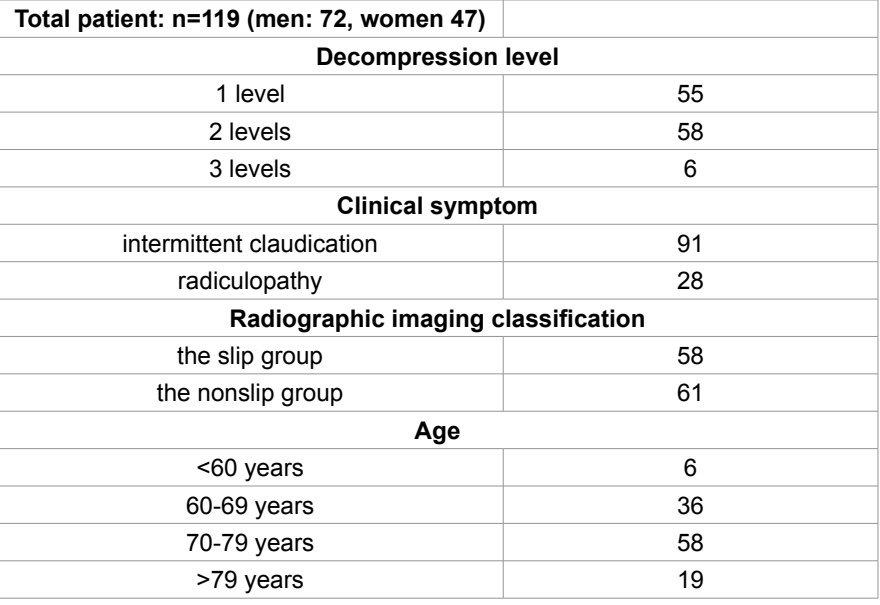

Table 1: Patient population

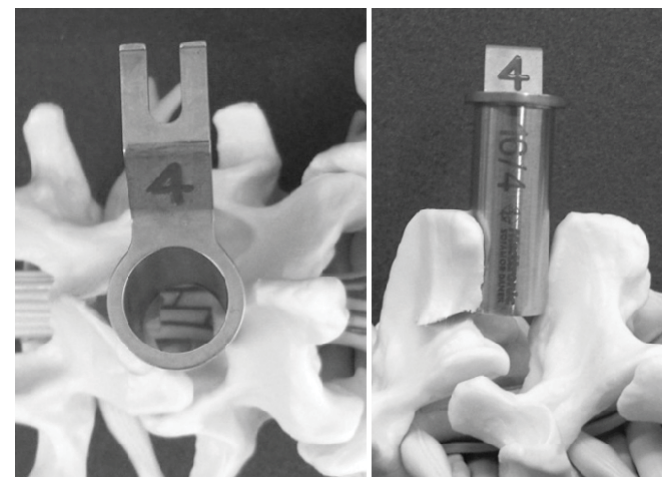

Figure 1: Lumbar spinal model demonstrating LSPSL in which an $18 \mathrm{~mm}$ diameter tubular retractor from the METRx microdiscectomy system is used for single-level decompression (left: dorsal view, right: lateral view).

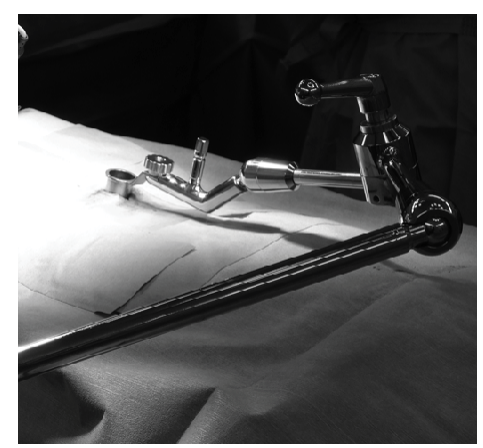

Figure 2: Intraoperative photograph demonstrating the tubular retractor mounted on the surgical table for single-level decompression.

\section{Surgical procedure}

The surgical procedure was performed in patients undergoing LSPSL as previously reported [8]. Briefly, after making a $20-\mathrm{mm}$ skin incision, the tip of the process was exposed. A caudal part of the process was cut longitudinally into the base using a straight chisel, and then followed with a 15-mm wide straight chisel in the same cutting line. The base was then cut using a curved chisel. While maintaining attachment to the rostral part of the process, the caudal part was opened in a "V" shape using a Cobb elevator. For single-level decompressions, an 18mm diameter tubular retractor from the $\mathrm{METRx}^{\mathrm{TM}}$ microdiscectomy system was inserted between the 2 halves of the process (Figures 1 and 2). For multi-level decompressions, a cervical retractor was used to open the 2 halves of the spinous process with 1 incision. After completing the microscopic decompression, a surgical drain was placed between the 2 halves of the process. Finally, the process was reconstructed with a nonabsorbable surgical suture. All patients were permitted to walk without a brace 1 day post-operatively.

\section{Clinical assessment}

Clinical improvement in neurologic symptoms was evaluated using the Japanese Orthopedic Association (JOA) score, which ranges from -6 to 29 [9]. Concomitant lower back pain was evaluated using the JOA score for symptomatic assessment of lower back pain (LBP-JOA score), which ranges from 0 to 9 [8]. Clinical improvement of pre-operative symptoms was evaluated using the JOA score, and the improvement rate was calculated using: (post-JOA - pre-JOA) / (29 - pre-JOA) x 100.

\section{Measurement of spinal instability after LSPSL}

Spinal instability after LSPSL was evaluated using a previously described method [8]. The slip rate was measured using radiographic imaging findings in the sagittal plane in the neutral position in the slip group ( $\mathrm{n}=58$; decompression levels, 62 ), using: slip distance (a) / length of the lower vertebral body $x 100$. In addition, the instability rate was measured using radiographic imaging findings in the sagittal plane at 3 positions: neutral, trunk extension, and flexion positions. First, each slip distance in the 3 positions was measured: the maximal (a-max) and minimal distance (a-min) were individually chosen, and the instability rate was defined as (a-max - a-min) / length of the upper vertebral body x 100 .

\section{Bony union of the split spinous process}

Bony union of the split spinous process at the region between the left and right halves of the process, and between the process and vertebral arch, was evaluated by using post-operative computed tomography (CT) imaging findings, as previously reported [8]. Overall, we evaluated post-operative CT in 73 patients (112 decompression levels).

\section{MRI signal change of the multifidus muscle after LSPSL}

MRI signal changes of the multifidus muscle after LSPSL was evaluated as previously described [8]. Eighty-eight patients (141 decompression levels) underwent post-operative MRI studies. The signal change of the multifidus muscle at the individual levels of the decompression by LSPSL were evaluated on T1-weighted imaging and T2-weighted imaging, and compared to those of pre-operative MRI findings. Scores were assigned as follows: 0 , same signal intensity; $1+$, slight increase; $2+$, moderate increase; or $3+$, strong increase of signal intensity of the multifidus muscle after surgery on T2-weighted imaging, compared with pre-operative findings. 


\section{Statistical analysis}

Differences between the 2 groups were analyzed using the MannWhitney U test. Statistical analysis was performed using the Sigma Plot 10.0 and Sigma Stat 3.5 for Windows (Microsoft Corp., Seattle, WA).

\section{Results}

Operating time for each patient was $196 \pm 70 \mathrm{~min}$. The mean operating time for single decompressions was $123 \mathrm{~min}$, and mean estimated blood loss was $55 \mathrm{~mL}$. Three major medical complications occurred, including 1 case of postoperative methicillin-resistant Staphylococcus aureus infection and 2 cases of minor spinal fluid leakage, as previously reported [8]. In addition, 3 follow-up surgeries were required after LSPSL, including transforaminal lumbar interbody fusion for LSS recurrence or for decompression level spinal instability. Five patients had spinal compression fractures either at the adjustment or non-adjustment level of the surgery (T12 level, 1; L1, 2; L2, 1; L3, 1) after LSPSL. One patient had a femoral neck fracture after LSPSL. In addition, 1 patient was treated with conventional laminectomy at the same level of the LSPSL, because of consequential lumbar disc herniation after LSPSL. One patient also had an onset of Parkinson disease after LSPSL. During the entire follow-up period after LSPSL, 3 patients died of adverse events unrelated to LSPSL.

JOA scores significantly improved at final follow-up $(23.8 \pm 4.3)$, compared with before surgery $(14.8 \pm 4.7)$ in all patients $(\mathrm{P}<.001)$. In particular, JOA scores significantly improved at the final follow-up, compared with before surgery in the intermittent claudication group (23.7 \pm 4.5 and $14.6 \pm 4.8$, respectively) and the radiculopathy group $(24.3 \pm 3.2$ and $15.7 \pm 4.2 ; \mathrm{P}<.001)$. JOA scores also significantly improved at the final follow-up, compared with before surgery in the nonslip group $(23.4 \pm 4.6$ and $14.8 \pm 4.7$, respectively; $\mathrm{P}<.001)$ and the slip group $(24.2 \pm 4.0$ and $14.9 \pm 4.7 ; \mathrm{P}<.001)$. LBP-JOA scores significantly improved in all patients at the final follow-up $(7.6 \pm 1.4)$, compared with before surgery $(6.3 \pm 2.0)(\mathrm{P}<.001)$. In particular, LBPJOA scores improved significantly at the final follow-up, compared with before surgery in the nonslip group $(7.5 \pm 1.6$ and $6.3 \pm 2.1$, respectively; $\mathrm{P}<.001)$ and the slip group $(7.8 \pm 1.3$ and $6.4 \pm 2.0 ; \mathrm{P}<$ $.001)$. No significant differences were observed in pre-operative JOA scores between the intermittent claudication and radiculopathy groups $(\mathrm{P}=.312)$ or in pre-operative LBP-JOA scores between the nonslip and slip groups $(\mathrm{P}=.884)$.

The overall improvement rate in all patients was $62.2 \pm 28 \%$. In addition, the improvement rate in the intermittent claudication and radiculopathy groups was $63.3 \pm 28.5 \%$ and $60.1 \pm 28.7 \%$, respectively; no significant differences were seen between groups $(\mathrm{P}=.621)$. Improvement rates based on age were $72.7 \pm 31.6 \%$ in patients younger than 60 years $(n=6), 72.1 \pm 24.2 \%$ in those $60-69$ years $(n=36), 59.5 \pm$ $28.7 \%$ in those $70-79$ years $(n=58)$, and $48.6 \pm 25.7 \%$ in those older than 79 years $(n=19)$. The improvement rate in patients younger than 80 years $(n=100)$ was $64.8 \pm 27.8 \%$. A significant difference in improvement rate was observed between patients younger than 80 years and those older than 79 years $(\mathrm{P}=.008)$.

Although no significant difference in the slip rate was seen preoperatively $(13.9 \% \pm 5.7 \%)$ and post-operatively $(15.7 \% \pm 6.7 \%)$ in the slip group, there was a tendency to slightly advance the slippage of the vertebral body after LSPSL $(\mathrm{P}=.108)$. No significant difference in the instability rate was seen pre-operatively $(6.2 \% \pm 3.8 \%)$, compared with post-operatively $(4.8 \% \pm 2.6 \%)(\mathrm{P}=0.061$, Table 2$)$.

The rate of bony union at the region between the left and right halves of the process was $97.3 \%(109 / 112)$, and the rate at a region

\begin{tabular}{|c|c|c|}
\hline & Current Study & Previous Study \\
\hline Slippage rate & & \\
\hline pre-LSPSL & $13.9 \% \pm 5.7 \%$ & $14.9 \pm 6.6 \%$ \\
\hline post-LSPSL & $15.7 \% \pm 6.7 \%$ & $15.4 \% 7.0 \%$ \\
\hline Instability rate & & \\
\hline pre-LSPSL & $6.2 \% \pm 3.8 \%$ & $5.8 \% \pm 3.7 \%$ \\
\hline post-LSPSL & $4.8 \% \pm 2.6 \%$ & $5.7 \% \pm 3.6 \%$ \\
\hline
\end{tabular}

Table 2: Slip rate and instability rate in the slip group both in the current study and the previous study.

between the base of the halves of the process and vertebral arch $79.5 \%$ (89/112). Union rates at a region between the base of the halves of the process and the vertebral arch in the nonslip and slip groups were $83.3 \%(45 / 54)$ and $75.9 \%$ (44/58), respectively. LBP-JOA scores at the final follow-up were $7.8 \pm 1.2$ in the group of cases with no floating of the spinous process (i.e., the base of the halves of the process and vertebral arch were united) $(n=53)$ and $7.6 \pm 1.3$ in the group of cases with floating of the spinous process $(n=20)$. No significant difference in LBP-JOA scores was seen between the groups $(\mathrm{P}=.0370)$. These results were similar to those in our previous study [8].

Post-operative MRI findings showed no change in signal intensity (0) of the multifidus muscle on T2-weighted imaging, compared with pre-operative findings in $89.4 \%$ of the decompression levels $(126 / 141)$. In addition, we found a slight increase in signal intensity $(1+)$ in $9.9 \%$ of the decompression levels 14 (14/141), and moderate increase $(2+)$ in $0.7 \%(1 / 141)$; no strong $(3+)$ signal intensity increase was detected. Among the 15 cases with a slight or moderate increase in signal intensity, 14 also demonstrated a slight signal increase on T1weighted imaging at the corresponding area of the multifidus muscle. In other words, T1- and T2-weighted imaging showed a slight increase in signal intensity of the multifidus muscle after surgery in $9.2 \%$ of all cases, indicating a small amount of fat infiltration. In the case in which a slight signal increase was only seen on T2-weighted imaging, we found an insignificant edematous change of the multifidus muscle. No significant differences in post-operative LBP-JOA scores were seen between patients with no signal intensity change $(7.7 \pm 1.4)$ and those with $1+$ or $2+$ signal intensity change $(7.7 \pm 1.2)$ of the multifidus muscle $(\mathrm{P}=.669)$. These results were similar to those in the previous study [8].

\section{Discussion}

In our previous study, we reported that LSPSL using a microscope and tubular retractor was one of the most promising minimally invasive laminectomy, because: it preserves most of the posterior supporting structures, without detaching the paravertebral muscles from the process; the incision is approximately $2 \mathrm{~cm}$ in single-level decompressions; patients' clinical symptoms were significantly improved after LSPSL; post-operative spinal instability did not occur after LSPSL; lower back pain was significantly improved after LSPSL in the non-slip and slip group; although the bony union rate in the slip group was relatively low, non-union was not associated with subsequent lower back pain; and aside from the small amount of fat infiltration in the multifidus muscle observed in a few cases, muscle degeneration of the paravertebral muscle was not obvious after LSPSL $[8,9]$. In the current study, we re-examined the results from our previous study and evaluated patients who underwent LSPSL with a follow-up greater than 24 months. We observed similar outcomes than in our previous study in terms of pre- and post-operative JOA scores, radiographic imaging findings to measure spinal instability after LSPSL, post-operative CT imaging to evaluate bony union of the split process, and MRI findings to find the muscular change of the multifidus muscle after LSPSL. 
These data suggest that clinical outcomes in patients who underwent LSPSL are stable and excellent.

We also examined the improvement rate of JOA scores based on age and found that not only it was $48.6 \pm 25.7 \%$ in patients who were older than 79 years at the time of the surgery, but also it was significantly lower than that of patients younger than 80 years $(64.8 \pm 27.8 \%)$. Our results seem sensible considering the aging effects associated with general physical activity. Nanjo et al. recently reported that the benefits and risks of decompression surgery in patients with LSS were similar in patients older than 80 years and those younger than 80 years, concluding that decompression surgery was a practical treatment even for elderly patients older than 80 years [10]. In addition, Yamashita et al. reported that older patients with LSS who undergo decompressive laminectomy have a greater risk for late recurrence of symptoms, especially 3 years post-operatively [11]. Although improvement rates observed in our study were relatively lower in patients older than 79 years, we encourage LSPSL among healthy elderly patients because their average life span has greatly increased. It should be noted that strict informed consent is required before surgery however, to inform this patient population of lower improvement rate with elder patients and avoid unnecessary disappointment associated with exceeding expectation. Furthermore, differential diagnoses, including disuse syndrome or intermittent claudication caused by chronic arterial occlusive disease, should be excluded because comorbidities are often present in elderly patients [12]. For those patients, care manager in the primary health care system may be able to support their activity of daily life [13].

Although no significant difference in the slip rate was seen preoperatively and postoperatively, we observed a tendency to advance the slippage of the vertebral body slightly after LSPSL in the slip group. In our previous study, the slip rate was $14.9 \% \pm 6.6 \%$ pre-operatively and $15.4 \% \pm 7.0 \%$ post-operatively, and the instability rate was $5.8 \% \pm 3.7 \%$ and $5.7 \% \pm 3.6 \%$, respectively [8] (Table 1 ). Since we did not observe this trend in the previous study, we believe that this novel phenomenon is likely associated with the long-term follow-up. Interestingly, we also found a tendency of a slight decrease in the instability rate, suggesting that spinal instability associated with spondylolisthesis may gradually improve after LSPSL in a time-dependent manner. These phenomena resemble the natural aging process of the lumbar instability, which was previously reported by Iguchi et al. [14] In their study, the authors showed that excessive sagittal translation, which was defined by radiographic imaging findings in the sagittal plane in the trunk extension and flexion positions, was relatively predominant in patients younger than 70 years [14]. In addition, they found that it gradually reduced with progressing disc degeneration although slip in the neutral position was frequently observed in elder age groups and increased with age [14]. We also found that slight advance of the slippage does not influence patients' clinical outcomes based on JOA and LBP-JOA scores. It is still controversial whether additional spinal fixation is essential in patients with LSS and low-grade spondylolisthesis [15]. It is commonly known that excessive facetectomy can cause spinal instability following laminectomy [16,17], and that the preserving technique of maximizing posterior supporting structures, including facet joint, is required in the surgical treatment of patients with LSS $[18,19]$. Additional spinal fusion is a gold standard option for patients with LSS. However, it is also associated with more complications than laminectomy alone, such as deep infection, implant fracture, bleeding, pseudarthrosis, adjacent segment disease, and occasional mortal neurovascular injury $[15,20]$. In addition, Ghogawala et al. recently reported that there is little value in adding fusion to decompression surgery, compared with decompression only, even with standard laminectomy [21]. Our study supports these results. Spinal fusion in addition to decompression should be avoided in patients with LSS and low-grade spondylolisthesis.

\section{Conclusion}

We conclude that the long-term outcome of patients who underwent LSPSL was a satisfactory minimally invasive decompression surgery, as previously reported. Although the improvement rate of the patients whose age at the time of the study was older than 79 years is relatively low, we suggest that this is acceptable consider the aging effects associated with general physical activity.

\section{Conflict of Interest}

No funds were received in support of this work. No benefits of any form have been or will be received from a commercial party related directly or indirectly to the subject of this manuscript. Portions of this work were presented in abstract form as proceedings at the Spine Across the Sea, North American Spine Society and Japanese Society for Spine Surgery and Related Research, Hawaii, USA., July 26, 2015.

\section{References}

1. Palmer S, Turner R, Palmer R (2002) Bilateral decompression of lumbar spinal stenosis involving a unilateral approach with microscope and tubular retractor system. J Neurosurg 97: 213-217.

2. Pao JL, Chen WC, Chen PQ (2009) Clinical outcomes of microendoscopic decompressive laminotomy for degenerative lumbar spinal stenosis. Eur Spine J 18: 830-836

3. Yagi M, Okada E, Ninomiya K, Kihara M (2009) Post-operative outcome after modified unilateral-approach microendoscopic midline decompression for degenerative spinal stenosis. J Neurosurg Spine 10: 293-299.

4. Hatta Y, Shiraishi T, Sakamoto A, Yato Y, Harada T, et al. (2009) Musclepreserving interlaminar decompression for the lumbar spine: a minimally invasive new procedure for lumbar spinal canal stenosis. Spine 34: E276-280.

5. Baghdadi YM, Moussallem CD, Shuaib MA, Clarke MJ, Dekutoski MB, et al (2016) Lumbar spinous process-splitting laminoplasty: A novel technique for minimally invasive lumbar decompression. Orthopedics 39: 950-956.

6. Kanbara S, Yukawa Y, Ito K, Machino M, Kato F (2015) Surgical outcomes of modified lumbar spinous process-splitting laminectomy for lumbar spinal stenosis. J Neurosurg Spine 22: 353-357.

7. Watanabe K, Hosoya T, Shiraishi T, Matsumoto M, Chiba K, et al. (2005) Lumbar spinous process-splitting laminectomy for lumbar canal stenosis. Technical note. J Neurosurg Spine 3: 405-408.

8. Nomura H, Yanagisawa Y, Arima J, Oga M (2014) Clinical outcome of microscopic lumbar spinous process-splitting laminectomy. J Neurosurg Spine 21: 187-194.

9. Izumida S, Inoue S (1986) Assessment of treatment for low back pain. J Jpn Orthop Assoc 60: 391.

10. Nanjo $Y$, Nagashima H, Dokai T, Hamamoto $Y$, Hashiguchi H, et al. (2013) Clinical features and surgical outcomes of lumbar spinal stenosis in patients aged 80 years or older: a multi-center retrospective study. Arch Orthop Trauma Surg 133: 1243-1248.

11. Yamashita K, Ohzono K, Hiroshima K (2006) Five-year outcomes of surgical treatment for degenerative lumbar spinal stenosis: a prospective observational study of symptom severity at standard intervals after surgery. Spine 31: 1484-1490.

12. Szpalski M, Gunzburg R (2003) Lumbar spinal stenosis in the elderly: an overview. Eur Spine J 12: S170-175.

13. Ciccone MM, Aquilino A, Cortese F, Scicchitano P, Sassara M, et al. (2010) Feasibility and effectiveness of a disease and care management model in the primary health care system for patients with heart failure and diabetes (Project Leonardo). Vasc Health Risk Manag 6: 297-305.

14. Iguchi T, Kanemura A, Kasahara K, Kurihara A, Doita M, et al. (2003) Age distribution of three radiologic factors for lumbar instability: probable aging process of the instability with disc degeneration. Spine 28: 2628-2633.

15. Deyo RA, Mirza SK, Martin BI, Kreuter W, Goodman DC, et al. (2010) Trends, 
Citation: Nomura H, Yanagisawa Y, Arima J (2016) Long-Term Outcome of Patients Undergoing Microscopic Lumbar Spinous Process-Splitting Laminectomy. J Spine 5: 343. doi: 10.4172/2165-7939.1000343

major medical complications, and charges associated with surgery for lumbar spinal stenosis in older adults. JAMA 303: 1259-1265.

16. Johnsson KE, Redlund-Johnell I, Udén A, Willner S (1989) Preoperative and postoperative instability in lumbar spinal stenosis. Spine 14: 591-593.

17. Johnsson KE, Willner S, Johnsson K (1986) Postoperative instability after decompression for lumbar spinal stenosis. Spine 11: 107-110.

18. Gunzburg R, Szpalski M (2003) The conservative surgical treatment of lumbar spinal stenosis in the elderly. Eur Spine J 12: S176-180.
19. Jönsson B, Akesson M, Jonsson K, Strömqvist B (1992) Low risk for vertebra slipping after decompression with facet joint preserving technique for lumbar spinal stenosis. Eur Spine J 1: 100-104.

20. Ghiselli G, Wang JC, Bhatia NN, Hsu WK, Dawson EG (2004) Adjacent segment degeneration in the lumbar spine. J Bone Joint Surg Am 86-A: 14971503.

21. Ghogawala Z, Dziura J, Butler WE, Dai F, Terrin N. et al. (2016) Laminectomy plus Fusion versus Laminectomy Alone for Lumbar Spondylolisthesis. N Eng J Med 374: 1424-1434. 